\title{
Normalización de la estrategia de enseñanza-aprendizaje Giras de Campo, para planificación y ejecución de sus tres momentos de ejecución: el antes, durante y después
}

Ing. Juan José Villavicencio Navarro Universidad Nacional Autónoma de Nicaragua, Managua UNAN-Managua, FAREM-Carazo jvilla0111@hotmail.com

Palabras clave: giras de campo, aprendizaje significativo, currículo, normalización, enseñanza-aprendizaje, competencias, objetivos.

\section{RESUMEN}

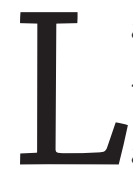
as Giras de campo es una de las principales estrategias didáctica que relaciona la teoría aprendida en el salón de clases con la práctica del campo laboral; En este artículo se pone de manifiesto aspectos que juegan un papel relevante para la aplicación de dicha estrategia, cabe señalar que son pocas las evidencias documentadas que se tienen sobre las realizaciones de la misma; y en la actualidad para muchos estudiantes y profesores se ha convertido en una simple excursión más que un proceso de aprendizaje significativo, por esta y otras razones, en las instituciones de educación donde se pone en práctica esta importante estrategia se deben de contar con instrumentos, que vienen a ser formatos que evidencien y permitan una adecuada planificación de ejecución durante los tres grandes momentos de la gira de campo que son el antes, el durante y el después; estos momentos se necesitan, más aún en aquellas universidades que hacen uso de la carpeta docente, donde las evidencias del actuar de los docentes son un componente esencial de la misma, puesto que contribuye a tener una fuente de información sobre los alcances que esta estrategia puede tener en el aprendizaje de los estudiantes, y sin obviar la perspectiva que estos mismos tienen hacia las giras de campo en las que han sido participe, pero que en muchas veces no se les toma en cuenta su perspectiva, la cual es muy importante conocer para la mejora continua en la aplicación de las giras de campo. 


\section{INTRODUCCIÓN}

La estrategia de enseñanza Giras de Campo, es una de las estrategias que permite establecer una vinculación estrecha entre la teoría y la práctica. Esta es usada en diferentes universidades del mundo y ha mostrado el alcance de excelentes resultados; sin embargo, diferentes autores como Echegaray (2013), López (s.f.), Rodríguez y Morales (2010) y Lossio (2004), que han escrito sobre la importancia de esta estrategia, han hecho referencia que para muchos docentes de educación superior, dicha estrategia se ha vuelto un paseo que carece de planificación y normalización en su ejecución, cabe señalar que, esta estrategia es realizada fuera del salón de clase, lo que indica que es responsabilidad del gremio académico y administrativo la correcta gestión de la misma, por consiguiente, para que esta estrategia tenga el alcance significativo, es necesario que se trabaje en conjunto con todos los que intervienen en la gestiones necesarias para llevar a cabo las Giras de Campo.

En el presente ensayo se dejan al descubierto los diversos pensamientos de autores que han hecho uso de las Giras de Campo como estrategia de enseñanza y que a la vez, han realizado investigaciones en el que el tema principal es esta estrategia; cabe señalar que, en su mayoría convergen en que, al carecer de planificación organizada, pierde efectividad, por lo que recomiendan los tres grandes momentos de ejecución de la misma: El antes, el durante y el después, lo cual permitirá que el estudiantado se sienta motivado, ante los presaberes y los nuevos conocimientos por descubrir, inclusive, cuando el objeto de estudio tiene estrecha vinculación a su perfil académico.

Es necesario recordar que las universidades viven en constantes transformaciones curriculares en el cual, siempre el objetivo primordial es hacer que el estudiante se dote de las competencias específicas que necesita tener como armas ante un mundo cambiante y desafiante, siendo esto el reto clave para el rol del docente universitario, quien debe seleccionar y ejecutar de la mejor manera posible, aquella estrategia de enseñanza - aprendizaje que permita alcanzar los objetivos propuestos en sus asignaturas.

\section{DESARROLLO}

\section{Diferentes concepciones de las Giras de Campo}

El Modelo Educativo de la UNAN-Managua (2011) menciona que las Giras de Campo son una estrategia importante para desarrollar en otro ambiente diferente al aula de clase; ponen en contacto al estudiante con la realidad mediante procesos cognitivos como la observación, la analogía, la descripción, el análisis, la síntesis, entre otros. Es decir, es un momento didáctico semiformal, porque requiere de la presencia efectiva del facilitador (el docente o equipo de docentes), en un ambiente que no es el salón de enseñanza (pág. 42). 
Para muchos docentes y estudiantes, las Giras de Campo son conocidas con diferentes nombres, como: giras técnicas, visitas a las instituciones, visitas de campo, recorrido, excursiones, trabajo de campo. Por tal razón, cuando se le pregunta a un docente qué es para él "Giras de Campo", probablemente en su definición tome en cuenta alguna de los nombres mencionados anteriormente. En consecuencia, se entenderán estos diferentes nombres como sinónimos.

Autores como Lossio (2004) afirman que la Gira de Campo es una estrategia didáctica que permite generar aprendizajes significativos en Geografía. Este autor lo diferencia como un método de enseñanza y resalta el interés particular que se debe tener en la comprensión de las ventajas de estos trabajos, pues coadyuvan a la retención, comprensión y uso activo del conocimiento; sin embargo, no solo obedece a esta concepción didáctica, en particular de la geografía y de las ciencias afines. Como bien menciona el autor Lossio, las Giras de Campo no solo permiten generar aprendizaje significativo en una asignatura en específico, sino en todas aquellas asignaturas que adopten esta estrategia como herramienta clave para el aprendizaje significativo del estudiante.

Rodríguez y Morales (2010) definen la Gira de Campo como una actividad académica, vivencial, programada, en la cual se integran la teoría y la práctica con diferentes modalidades de evaluación, que "pueden ser de aplicación, de observación, o análisis de campo".

Enla definición de estos autores selogra observarque estáinmersala palabra "programada", esto indica que la estrategia Giras de Campo tiene que ser planificada previamente, y no ser llevada a cabo de manera espontánea, puesto que podría perder el enfoque del potencial logro de los objetivos previamente planteados, en el caso de haberse programado dichos objetivos, puesto que al carecer de programación, probablemente hasta se carezca de estos.

Las concepciones de la Gira de Campo son muchas, desde el punto de vista de diferentes autores que han estado inmersos en este tema, pero al final, todos convergen en el mismo punto, en que ella es una estrategia que permite relacionar la teoría con la práctica y que debe tener una adecuada planificación.

\section{Giras de Campo, ¿estrategia o recurso?}

Los docentes noveles tienden en lo general a confundir una serie de conceptos, entre ellos, estrategias didácticas y recursos didácticos; sin embargo, cada uno de ellos tiene una función específica, lo cual no indica que sean contradictorios, sino más bien, se complementan para facilitar el proceso de enseñanza-aprendizaje, como se puede apreciar en las siguientes definiciones:

En el campo educativo, se denomina estrategia didáctica a la planificación de un grupo de actividades y herramientas para dar respuesta a las necesidades del conjunto de las personas y 
los objetivos que estas se han propuesto. El propósito final se centra en facilitar el aprendizaje; es la forma en la que él o la docente garantiza que el estudiante integre los contenidos (Carbonell, 2004).

Por su parte, los recursos didácticos se definen como instrumentos mediadores o facilitadores entre él o la estudiante y el contexto o la realidad. Ya sea un objeto natural, una pizarra, el ordenador o la propia explicación del docente. El recurso didáctico es ese elemento que ayuda a obtener los conocimientos (Bedmar, 2009). Cabe decir que, el recurso didáctico no es el que genera el conocimiento, pero sí facilita los procesos de enseñanza-aprendizaje que desempeña el docente.

Como bien se puede observar en las definiciones de ambos autores, la Gira de Campo es una estrategia que debe valerse de recursos para su realización, recursos tan importantes como el medio de transporte, el cual es fundamental para la realización de esta estrategia, de tal manera que, resulta necesario la existencia de medios de transporte que estén a disposición para llevar a cabo esas Giras de Campo.

\section{Las Giras de Campo, una realidad preocupante}

Según el autor López Martín (2007), en su artículo "Las salidas de campo, mucho más que una excursión", plantea que es un hecho constatable que cada vez se sale menos al campo con el alumnado y que, cuando se hace, las salidas son meras excursiones. Agrega que algunas de las posibles causas que han contribuido a ello son las siguientes:

- El miedo del docente a las responsabilidades que se asumen en este tipo de actividad extraescolar.

- El bajo grado de satisfacción del docente en estas actividades.

- La falta de formación de los docentes en aspectos prácticos de campo, así como su desconocimiento del entorno.

- La falta de una buena planificación y metodología que impide un buen aprovechamiento de la actividad.

Entre los aspectos más negativos, algunos estudiosos coinciden en que no disponen de suficiente tiempo para preparar las salidas y que resulta muy complicado disponer de otros docentes que se impliquen en la actividad. Echegaray (2013) menciona lo costoso que resulta conseguir algunos permisos en la administración de los centros de estudio para realizar salidas de campo específicas.

Atendiendo a lo que dicen los autores, queda reflejado que, son muchos los docentes que observan grandes dificultades para realizar las salidas de campo, respecto al tiempo que es 
necesario dedicar y al problema que supone tener que disponer de más docentes. Esta puede ser la principal razón por la que el grado de satisfacción general de ellos no sea muy alto. Sin embargo, Echegaray (2013) afirma que, las salidas de campo resultan muy satisfactorias tanto para el docente como para el estudiante.

Tal a como se logra observar en las citas de estos autores, las Giras de Campo no son muy frecuentes y las razones son muchas; sobre la base de esto es necesario reflexionar y hacer conciencia que es necesario identificar la causa de la raíz de esto y volver lo negativo en positivo.

Puede resultar interesante y propicio para las instituciones de educación superior y aún más para los docentes, normalizar la realización de la Giras de campo, mediante el uso de instrumentos estandarizados que presenten flexibilidad para todas aquellas asignaturas que hacen uso de esta estrategia didáctica tan importante que se lleva a cabo fuera del aula de clase y que requiere de control, esto podría contribuir en el rol del quehacer docente, donde se podrá contar con documentaciones que sirvan de evidencias docentes, y por ende, se puedan observar las diferentes Giras de Campo que se han llevado a cabo en la institución y a la vez puedan ser bases para futuras giras. Contar con evidencias de ellas, vendría a ser parte fundamental para el apartado de evidencias docentes, que es un apartado fundamental en la carpeta docente que se pone de manifiesto en muchas instituciones de educación.

\section{Cómo sacar el máximo rendimiento a una Gira de Campo}

Según el estudioso López Martín (2007), para obtener un buen aprovechamiento de las Giras de Campo es muy importante realizar una buena planificación, utilizar una metodología apropiada y dedicarle el tiempo necesario a cada una de las tareas programadas. El estudiante debe, en todo momento, conocer en qué fase de la Gira de Campo se encuentra, cuál es el objetivo de la misma. Estas giras deben ser desarrolladas de forma contextualizada, con un conjunto de actividades de enseñanza-aprendizaje articulado entre sí, que contribuyan a conectar los conocimientos del estudiante antes, durante y después de la salida. Los materiales elaborados para esa salida deben estar pensados para ejercitar destrezas (observar, anotar, dibujar, entre otras) pero también, para fomentar la reflexión, incentivando al estudiante a exponer sus ideas y ejercitar capacidades. Antes de la salida de campo, el docente explicará su contenido (descripción general del lugar), cada una de las fases de que consta, descripción del itinerario, tipo de itinerario, inicio, final, distancia, duración y paradas que se van a realizar, las tareas que hay que realizar y los objetivos que se pretenden alcanzar. Es importante la buena organización de la salida de campo, tomar las precauciones y medidas de seguridad necesarias, contar con las fichas de recogida de datos, cuadernos de campo, cámaras fotográficas y todo aquello que permita la recogida de información. 
Echegaray (2013) sugiere aspectos que deben definirse a la hora de establecer el diseño de una salida de campo:

- Los objetivos que se pretenden alcanzar

- Las necesidades del estudiante

- Las características del estudiante

- Los medios y materiales de los que se dispone

- Los resultados que se pretenden obtener

- La forma de integrar lo aprendido en la salida de campo con la materia impartida en el aula

Como menciona el autor, es evidente que no se deben llevar a cabo las Giras de Campo sin una planificación adecuada y mucho menos sin hacerle saber el proceso a los estudiantes, iniciando por la sensibilización, donde la gira debe darse a conocer al estudiante en uno de los primeros encuentros en que se imparte la asignatura, esto debido a que ella debe estar contemplada en el plan semestral, y entre el primer anuncio y el día de la gira debe haber una constante sensibilización del estudiante, que a la vez se convierta en una motivación, esto ayudará a comprender al estudiante que la Gira de Campo como tal, no es el fin de la clase, sino una estrategia planificada que le ayudará a abstraer de la realidad la teoría que estudia en clase o por su cuenta.

La formulación de los objetivos es fundamental en las Giras de Campo, cuya redacción debe estar acorde al perfil académico del estudiante, cabe resaltar, que el sitio donde se lleve a cabo esta gira debe estar vinculado a los objetivos planteados y sobre todo, al perfil del futuro profesional, sin olvidar la previa constatación del sitio a visitar, en el cual se debe verificar que cuente con normas de seguridad para los estudiantes, ya que de lo contrario, tiende a causar incertidumbre tanto en las autoridades universitarias como en las familias de los estudiantes.

\section{Las Giras de Campo desde la perspectiva del docente y estudiantes Perspectiva docente:}

Mucho se ha dicho en la literatura pedagógica que, los propósitos de la enseñanza deben extenderse más allá de los contenidos curriculares circunscritos al aula de clase, por tanto, existe la necesidad de adquirir y desarrollar múltiples capacidades en distintos contextos para afrontar las demandas de la sociedad actual. En este sentido, la Gira de Campo es un buen ejemplo, pues constituye una convivencia circunstancial contextualizada fuera de la institución educativa cuya implementación requiere de una enseñanza estratégica (Lara, 2011). 
En el caso de los docentes, sus perspectivas se orientan por las siguientes reflexiones producto de sus experiencias de campo, a partir de las cuales se obtienen datos representativos que permiten dar cuenta de las creencias implícitas de los docentes, lo cual orientan la acción transformadora de sus prácticas. El docente debe tener sumo cuidado al planificar una Gira de Campo, en un principio, debe indagar lo que el estudiante conoce del contexto en el cual se realizará el trabajo, iniciar una fase de documentación con material de apoyo, luego debe facilitar los materiales a utilizar, entre ellos, la guía de campo que es vital porque allí se establecen objetivos, actividades, paradas, recursos, evaluación, entre otros (Lara, 2011).

Tal como se observa en las citas de esta autora, la perspectiva de las Giras de Campo tendrá una panorámica diferente para los ojos de cada docente, siendo importante fomentar en ellos que es necesario planificar la gira y sobre todo, encender la chispa del entusiasmo y curiosidad de los estudiantes por el aprendizaje, y más aún, al mostrarle una guía de campo previamente elaborada, que permita acercar al estudiante al contexto bajo estudio y que lo haga sentir parte de este.

\section{Perspectiva del estudiante:}

Las Giras de Campo, desde la perspectiva del estudiante, no han sido muy investigadas y documentadas, a tal medida que no se encuentra información en la Web al respecto, lo cual resulta ser interesante para realizar investigaciones que permitan obtener datos con respecto a las giras desde la perspectiva del estudiante.

\section{Momentos de la Gira de Campo}

Estas Giras asumen un esquema con tres momentos importantes, de acuerdo a las publicaciones del Centro Nacional para el Mejoramiento de la Enseñanza de la Ciencia, CENAMEC (s.f., pág. 5) en las que plantean que dentro de las actividades que se deben considerar al momento de planificar una salida de campo, destacan: Antes (trabajo de preparación previa); durante (actividades a desarrollar durante la salida) y después (trabajo de análisis, discusión y evaluación).

\section{Antes de las Giras de Campo:}

El docente debe prever algunas consideraciones entre las que destacan: (a) Seleccionar el (los) objetivo(s) o la(s) competencia(s) que se desea(n) desarrollar, reforzar o ampliar. (b) Buscar información referente al área de estudio (datos históricos, clima, suelos, vegetación, entre otras que el docente considere importante). Estos se constituyen en datos secundarios obtenidos de fuentes indirectas. (c) Realizar una visita de reconocimiento para efectuar el itinerario del trabajo. Se cree necesario hacer un bloqueo de la zona (inspección previa) en la cual se observen las áreas disponibles para ejecutar las actividades, verificar los espacios de riesgos, 
vías de escape, préstamos de servicios, verificación de horarios de visitas habituales (depende del lugar), limitaciones de la zona. (d) Efectuar los trámites administrativos (permisología para los estudiantes ante los padres y representantes y las autoridades respectivas; en caso de ser necesario, realizar la solicitud previa del permiso para la visita; solicitud de la exoneración del pago o aplicación de la promoción por ser institución escolar; solicitud de la visita guiada; contratación o disponibilidad del transporte u otro que el docente considere). (e) Establecer un cronograma de trabajo y la distribución de las tareas por cada participante de la actividad. Además, es importante elaborar un material didáctico con breves explicaciones, un guion de preguntas, algunas orientaciones, entre otras. (f) Negociar las normas de disciplina y dialogar acerca del propósito de la actividad, sus beneficios e importancia. Conversar sobre las normas de seguridad y los riesgos en caso de infringir las mismas. (g) Verificar el estado de salud físico de cada participante. Considerar aquellas personas que presentan alguna enfermedad, en la cual, necesariamente deben consumir medicamentos. (h) Verificar que algún miembro o acompañante posea conocimientos sobre los primeros auxilios. También es necesario determinar el recurso didáctico requerido. Estos deben ser específicos de acuerdo a su uso, lugar de visita, la duración y objetivos que se persiguen con las Giras de Campo.

Inicialmente, se puede emplear una variedad de fuentes de información: libros, referencias electrónicas, folletos editados, guías especializadas, materiales de promoción, material audiovisual, entre otros. También se pueden utilizar los archivos, censos y estudios locales. Todo este material permitirá obtener suficiente información sobre la localidad, tener una visión global sobre sus condiciones, sus límites, nivel de seguridad, vialidad, organización, funcionalidad, estructura, zonas recreativas y de esparcimiento, zonas de trabajo, tipos de visitas (guiadas, semi-guiadas, no guiadas), sitios históricos o relevantes y otros.

Dentro de las consideraciones por tomar para el provecho académico de una Gira de Campo, López (s.f.) indica la importancia de que el estudiante conozca cada una de las fases de la actividad, por lo que recomienda la explicación del contenido antes o al inicio de la gira, la descripción del lugar, la distancia, las generalidades logísticas, los materiales necesarios, las tareas por realizar, los protocolos científicos y los objetivos que se pretenden describir. De esta forma, al aclarar una serie de aspectos previos, los estudiantes pueden ejercer con destreza las habilidades requeridas, así como fomentar los periodos de reflexión, lo cual va a incentivar al estudiante a exponer sus ideas y ejercer sus capacidades.

De las consideraciones anteriores, se puede decir que, las Giras de Campo conllevan una serie de actividades previas que necesitan de inversión de tiempo por parte del docente; probablemente, en no todas los Giras de Campo se tenga que hacer uso de todos estos requisitos previos, sino que estará en dependencia del contexto de estudio, el perfil académico de la carrera, y la duración misma de la Gira de Campo. 
Según en el I Encuentro, Vygostski y su legado en la investigación latinoamericana de Ulate de Brooke (2014), en el proceso didáctico de una gira de campo se dan varios momentos que se deben contemplar para su debida planificación. Añade que las giras de campo se pueden dividir en fases o etapas, tales como:

- Fase inicial: se den instrucciones, material impreso, conocimientos previos, objetivos, lineamientos generales, guía, entre otros.

- Fase de ejecución: se genera la práctica, para lo cual se debe contar con una guía de seguimiento, rúbrica e instrumentos que permitan describir lo observado y lo medido.

- Fase de recopilación de la información o elaboración del informe.

- Fase de socialización de la experiencia: se comparte la experiencia. Puede ser por medio de los recursos tecnológicos disponibles en la institución como las plataformas de aprendizaje. Para ello, se recurre a foros, blogs o bien, presencialmente en una discusión o realimentación en grupo (tutoría).

Son varios los requerimientos para las giras de campo mencionados en este encuentro, entre ellos, los estudiantes requieren documentos que los guíen instruccionalmente, antes, durante y después de la gira de campo. De igual forma, en esos materiales de instrucción se debe incluir información relacionada con las rúbricas de evaluación, guías para la presentación de informes, reglamentos y normativas, protocolo de comportamiento, aspectos de seguridad a contemplar, equipo requerido, materiales, orientaciones, tipo de ropa a llevar, advertencias de seguridad (normativa internacional y médica), metodología en la entrega de informe, requisitos y prerrequisitos, aspectos de primeros auxilios, consideraciones de salud, problemas psicológicos y explicación de las etapas a realizar.

\section{Durante las Giras de Campo:}

(a) Organizar los equipos de trabajo en función de las actividades a realizar, de los instrumentos y equipos disponibles y del espacio del que se dispone para el desarrollo de dicha actividad. (b) Realizar las observaciones cualitativas del área, en función de los objetivos que se plantea en el cronograma de actividades. (c) Realizar mediciones cuantitativas (si es el caso) y tomar las anotaciones pertinentes para su posterior análisis en clase. (d) Realizar recorridos con guía turístico (visita guiada, opcional). (e) Aplicar entrevistas o encuestas a personajes locales, historiadores, cronistas, autoridades. (f) Realizar grabaciones, tomar fotografías, levantar planos, realizar dibujos, trazar figuras y bocetos, entre otras. Especial importancia adquieren los datos primarios recogidos en este momento, pues posibilita la construcción y reconstrucción del conocimiento, la verificación de las hipótesis, la validación y contrastación de las teorías. Durante el desarrollo de las Giras de Campo, es necesario verificar las normas de disciplina y de seguridad, 
entregar el material didáctico que oriente su ejecución y brindar algunas consideraciones para la elaboración del informe final (Delgado, 2012, pág.174).

Resulta muy importante resaltar lo que este autor presenta, de manera que de nada sirve contar con todo un material didáctico preparado con anticipación, si este no es entregado al estudiante antes de realizar la Gira de Campo; desde otra perspectiva, cabe señalar que, es muy necesario que el docente explique todos aquellos documentos que tengan que llenarse en la realización de las Giras de Campo y que el estudiante sea partícipe de ello.

En esta modalidad de trabajo, el estudiante es la parte activa, es él el protagonista. El docente, durante la realización del recorrido, es el único elemento pasivo y solo en contadas ocasiones interviene conduciendo u orientando. Previamente a la realización de las Giras de Campo se ha debido proporcionar la información y los elementos necesarios para que el estudiante sea capaz de llegar a cubrir aquellos objetivos que se pretenden (Ramírez y Lozada, 2011).

\section{Después de las Giras de Campo:}

(a) Realizar el análisis de los datos obtenidos durante las mediciones en campo. (b) Realizar con los estudiantes una discusión dirigida, acerca de los aspectos más relevantes desarrollados durante el trabajo, revivir la experiencia, evocar momentos y conocimientos. Además, de conocer las diferentes interpretaciones sobre los significados construidos. (c) Realizar una valoración de los nuevos aprendizajes en términos de conocimientos construidos o reconstruidos. (d) Los estudiantes deberán realizar un informe que contemple el objetivo de la actividad, el análisis de los datos, el itinerario y cronograma de trabajo, la metodología utilizada, los resultados obtenidos, las conclusiones y la importancia de la actividad realizada (Delgado, 2012, pág.175).

Al finalizar las visitas se elaborará un informe escrito del trabajo efectuado en forma conjunta por el grupo y su docente referente. Se podrá además, comunicar los resultados buscando modalidades más informales. Puede ser entre otras, la elaboración de una obra creativa utilizando lo lúdico como herramienta para mostrar los mismos. La importancia de los datos recabados y las minutas de campo, radica en que esta es la etapa de cierre en la cual se harán los análisis y discusión de todos (Ramírez y Lozada, 2011).

En relación con este último momento sobre las Giras de Campo, es necesario conocer el alcance que tuvo esta estrategia, que mejor descubrirlo a través de la percepción de los estudiantes, para esto, los diversos autores coinciden que se debe hacer uso del informe escrito analítico - reflexivo, en el que el estudiante ponga de manifiesto sus logros, descubrimientos, observaciones y aprendizaje alcanzado. 
A lo largo de los planteamientos hechos, el lector puede notar que la Gira de Campo es una estrategia que aparentemente conlleva mucho esfuerzo de realización, no obstante, cuando el docente incursiona con esta estrategia, esto probablemente resulte ser cierto, sin embargo, con la práctica de la misma se va afinando detalles, hasta el momento en que el docente puede decir: "la realización disciplinada, planificada, organizada, controlada y normalizada, termina resultando ser atractivo, fácil, interesante y sobre todo, enriquecedor para el aprendizaje”.

\section{CONCLUSIÓN}

Es recomendable realizar las Giras de Campo a inicios de impartir la asignatura o a nivel intermedio de la misma, puesto que si se realiza al final, los estudiantes podrían interpretarla como "la despedida del semestre".

Finalmente, es necesario recalcar que, la estrategia Gira de Campo es aplicada no solo para una asignatura en específico, sino que, a pesar de que sea normalizada, podrá ser aplicada en cualquier asignatura, siempre y cuando los objetivos propuesto tengan estrecha vinculación con el sitio prospecto en donde se llevará a cabo dicha estrategia; por consiguiente, es necesario tomar consciencia y aceptar que para muchos educadores no es fácil aceptar la idea de trabajar con una estrategia que disponga de instrumentos normalizados, es decir, una estrategia debidamente planificada en sus tres grandes momentos de ejecución: antes, durante y después; en el que cada uno de estos momentos contempla un trabajo que para muchos es arduo, y para otros es muy interesante y enriquecedor, y he aquí, la percepción que todo docente debe tener, puesto que la planificación, organización, dirección y control permitirán que una estrategia que no se ejecuta en el salón de clase, sea llevada a cabo de la mejor manera posible, y esto permitirá que el principal beneficiado sea el estudiante, puesto que al realizar con ellos Giras de Campo con instrumentos normalizados, logrará que el estudiantado se acerque a su potencial campo laboral, y sobre todo, a la vinculación de la teoría impartida en el salón de clase, con el entorno contextual, donde el futuro profesional pondrá en práctica lo aprendido en su vida estudiantil.

\section{REFERENCIAS BIBLIOGRÁFICAS}

Atencio Ramírez, Maxula, Gouveia, Edith Luz, Lozada, Joan M. (2011). Las

Bedmar, J. (2009). Recursos didácticos en el proceso de enseñanza-aprendizaje. Revista digital para profesionales de la enseñanza.
Carbonell, J. (2004). Estrategias institucionales para el logro de aprendizajes significativos en las cátedras de computación e información. Universidad Interamericana para el desarrollo.

CENAMEC. (s.f.). El trabajo de campo. Disponible en: https://bit.ly/2qCPmTT 
Delgado, R. (2012). Las Giras de Campo como estrategia pedagógica integradora. Universidad Pedagógica Experimental Libertador. Caracas. Venezuela. Revista de Comunicación de la SEECI. (Julio 2013). Año XVII (31), 156-183 ISSN: 15763420 DOI: https://dx.doi.org/10.15198/ seeci.2013.31.156-183156156

Echegaray, K. (2013). Salidas de campo como recurso en la enseñanza de ciencias en bachillerato, una propuesta para ciencias de la tierra y medioambientales del 2do curso. Bilbao, España. UNIR.

López, J.A., (s.f.). La salida de campo: mucho más que una excursión. Recuperado de: www. educarm.es/templates/portal/.../11/22_ salidasdecampo.pdf

López, Martín. (2007). Las salidas de campo: mucho más que una excursión. Disponible en: https://www.researchgate.net/ publication/39222574_Las_salidas_de_ campo_mucho_mas_que_una_excursion

Lossio, O. (2004). Las Giras de Campo enespacios urbanos como propuesta de enseñanza facilitadora de aprendizajes significativos en geografía”. XXIV Semageo. Universidad Federal de Santa Catarina. Departamento de Geociencias. Resúmenes de mini discursos. Disponible: http://www.cfh. ufsc.br/ gcn/semageo\%2025/resumo21. htm
Rodríguez Lara, Juana. (2011). El Aprendizaje Colaborativo en el Laboratorio de Ciencias: Una estrategia didáctica significativa. Disponible en: https://bit.ly/2HpT9xA

Rodríguez, M. \& Morales, F. (2010). Propuesta de normas y reglamento para las giras y prácticas de campo del Programa de Protección de Manejo de Recursos Naturales, desde la Perspectiva de la Administración Educativa. (Tesis de Maestría). San José, Costa Rica: Universidad Estatal a Distancia.

Ulate de Brooke, Rosita. (2014). I Encuentro, Vygotsky y su legado en la investigación latinoamericana. Disponible en: https://inie.ucr.ac.cr/investigacion/ documentos.../category/10-i-encuentrovigotski

Universidad Nacional Autónoma de Nicaragua, Managua, (2011). Modelo Educativo, Normativa y Metodología para la planificación curricular 2011. 